\title{
Motivo de recusas em pesquisa: um estudo qualitativo no seguimento do estudo Fragilidade em Idosos Brasileiros - FIBRA-RJ
}

\author{
Pricila Cristina C. Ribeiro, ${ }^{1 *}$ Maria Angélica Sanchez, ${ }^{2}$ Débora M. Coelho, ${ }^{2}$ Roberto A. Lourenço ${ }^{2}$
}

\section{Resumo}

Estudos epidemiológicos com a população que envelhece são fundamentais para auxílio nas políticas públicas de saúde na atualidade. O inadequado delineamento destas pesquisas com perdas amostrais e a não representatividade da população-alvo implicam em estimativas imprecisas dos desfechos de saúde estudados. Assim, pesquisadores demandam estratégias de captação de sua população de estudo que garantam o mínimo de recusas e perdas amostrais em seus estudos longitudinais. $\mathrm{O}$ objetivo deste estudo foi compreender os motivos de recusa entre idosos selecionados para a participação no estudo de seguimento da Fragilidade em Idosos Brasileiros-Rio de Janeiro. A população de estudo foi composta de 67 indivíduos que, por motivo de recusa, não participaram da etapa de seguimento. Estes correspondem a 7,9\% da amostra da linha de base. Foi conduzida uma análise de conteúdo do tipo categorial temática para o tratamento do relato apresentado como motivo da recusa dos idosos ou de seus informantes. Foram obtidas $53,7 \%$ de recusas por parte dos próprios idosos e $46,3 \%$ por parte do informante. Os núcleos figurativos obtidos para descrever os motivos de recusa mais frequentes entre os próprios idosos foram a falta de credibilidade e desconfiança da pesquisa, e os mais frequentes entre seus informantes foram falta de tempo, cuidado médico vigente e doença do idoso. Estes resultados apontam que pesquisas em saúde do idoso devem se organizar para que as perdas de participantes não ocorram de forma sistemática em decorrência dos prejuízos da saúde neste grupo populacional. Além disto, a recusa por falta de credibilidade e a desconfiança em relação à pesquisa apontam na direção de que estudos na área da saúde precisam reduzir o distanciamento entre seus resultados de pesquisa e o cotidiano da população-alvo.

Descritores: Pesquisa qualitativa; Envelhecimento; Idoso.

\section{Abstract}

\section{Reason for refusals in research: a qualitative study in the follow-up of Fragility in Elderly Brazilian - FIBRA-RJ}

Epidemiological studies with the aging population are fundamental to aid in public health policies today. Improper design of these studies with sample loss and not representative of the target population implies inaccurate estimates of health outcomes studied. Thus, researchers need strategies to capture their study population to ensure minimal refusal and sample losses in their longitudinal studies. To understand the reasons for refusal of selected subjects for participation in the
1. Faculdade de Filosofia e Ciências Humanas. Universidade Federal de Minas Gerais. Belo Horizonte, MG, Brasil.

2. Laboratório de Pesquisa em Envelhecimento Humano, Faculdade de Ciências de Médicas, Universidade do Estado do Rio de Janeiro. Rio de Janeiro, RJ, Brasil.

*Endereço para correspondência:

E-mail: priccr@gmail.com

Revista HUPE, Rio de Janeiro, 2015;14(4):6-12

doi: $10.12957 /$ rhupe.2015.20065

Recebido em 22/10/2015. Aprovado em 08/12/2015.

follow-up study of Frailty in Brazilian Older People Study -Rio de Janeiro. The study population was composed of 67 individuals that did not attend the follow-up due to refusal, they constitute $7.9 \%$ of the sample from the baseline. The thematic categorial content analysis was conducted for the treatment of the report of the elderly or their informants presented as a reason for refusal. It has been obtained $53.7 \%$ of refusals from the elderly themselves and $46.3 \%$ from the informant. The figurative cores obtained to describe the most common reason for refusal among the elderly were the lack of credibility and mistrust of the research, and the most common core between his informants were lack of time, the current medical care and disease of the elderly. These results show that research in the health of the elderly should be organized so that the losses of participants do not occur systematically as a result of health damage in this population group. In addition, the refusal due to lack of credibility and mistrust of research points that research in health need to reduce the gap between theresults and the daily life of the target population.

Keywords: Qualitative research; Aging; Elderly.

\section{Resumen}

Motivo de la denegación en investigación: un estudio cualitativo en el seguimiento del Estudio Fragilidad en los ancianos brasileños - FIBRA-RJ

Estudios epidemiológicos de la población adulta mayor son fundamentales para ayudar en las políticas de salud pública en la actualidad. El diseño inadecuado de estas encuestas con pérdida de la muestra y no representativo de la población objetivo implica estimaciones inexactas de los resultados de salud estudiados. Por lo tanto, los investigadores requieren estrategias de captación de su población de estudio para 
garantizar un mínimo de negaciones y pérdidas de muestra en sus estudios longitudinales. Objetivo: Comprender los motivos de denegación de los adultos mayores seleccionados para participar en el estudio de seguimiento de Fragilidad en Adultos mayores brasileños de Rio de Janeiro.

Método: La población de estudio estuvo compuesta de 67 individuos que con el motivo de denegación no asistió a la etapa de seguimiento, éstos constituyen el 7,9\% de la muestra de la línea de base. Se realizó un análisis de contenido del tipo categoría temática para el tratamiento del informe presentado como motivo de la denegación de las personas mayores o de sus informantes. Resultados. Se obtuvieron 53,7\% denegaciones por parte de los propios adultos mayores y $46,3 \%$ de los informantes. Los núcleos figurativos obtenidos para describir los motivos de denegación más frecuentes entre los adultos

\section{Introdução}

O envelhecimento populacional é um fenômeno crescente. Em projeção populacional, feita pelo Instituto Brasileiro de Geografia e Estatística (IBGE), ${ }_{1}^{1} \mathrm{o}$ percentual da população idosa será maior que $22,71 \%$ na população total em 2050. Em 2008, essa parcela da população correspondia a 6,53\%, enquanto que crianças de zero a 14 anos ocupavam $26,47 \%$ do percentual total da população. Em decorrência desse processo de envelhecimento, nas últimas décadas, os perfis de saúde da população também passaram por transformações, como o aumento da incidência e prevalência das doenças cronicodegenerativas. Essas, por sua vez, elevam o número de pessoas susceptíveis a uma cadeia de eventos adversos, ${ }^{2}$ como perda funcional, redução da qualidade de vida do paciente e de seu núcleo familiar. Nesse contexto, investigações epidemiológicas sobre os agravos que acometem esse segmento etário são imprescindíveis para se pensar políticas públicas em saúde do idoso.

Nos últimos anos vem ocorrendo um aumento significativo de incentivos e produção da pesquisa gerontológica, ${ }^{3,4}$ sejam estudos de prevalência, sejam acompanhamentos longitudinais voltados para a incidência e a identificação dos fatores de prevenção ou de risco para as patologias associadas ao aumento da idade. Contudo, para que esses estudos forneçam evidências capazes de viabilizar a redução da incidência dessas patologias e de seus prejuízos e, consequentemente, de impactar a gestão de gastos em saúde pública, é preciso fazer investigações de base populacional com criteriosa seleção da amostra e da equipe de pesquisa responsável pela coleta de dados. Nessa direção, são muitos os percalços encontrados para a adequação metodológica. mayores fueron la falta de credibilidad y la desconfianza en la investigación, y los más frecuente entre sus informantes fueron la falta de tiempo, la atención médica actual y enfermedad de los adultos mayores.

Discusión: Estos resultados apuntan a que la investigación en la salud de los adultos mayores debe organizarse de manera que las pérdidas de los participantes no se produzcan de manera sistemática como resultado de daños a la salud de este grupo poblacional. Además, la denegación por falta de credibilidad y desconfianza con relación a la investigación apunta en dirección a que los estudios en el área de salud necesitan reducir la brecha entre los resultados de la investigación y el día a día de la población objetivo.

Palabras clave: Investigación cualitativa; Envejecimiento; Ancianos.

Dentre os entraves, destaca-se a dificuldade de adesão dos potenciais sujeitos de pesquisa. Os auxiliares de pesquisa que atuam diretamente no recrutamento da amostra, ainda que bem treinados, enfrentam problemas, como a falta de credibilidade nos projetos de pesquisa e até desconfiança de quem está entrando em contato para coletar dados.

$\mathrm{Na}$ área da saúde, as discussões das estratégias para melhora da captação de participantes de pesquisa são, em geral, acerca das regras para remuneração que, no contexto brasileiro, resumem-se ao ressarcimento de despesas como transporte e alimentação do participante e de seus acompanhantes. ${ }^{5}$ Contudo, a remuneração da população de estudo pode ser inviável em estudos populacionais, dado o aumento que acarreta no custo das pesquisas e no risco adicional de viés de seleção. Assim, apesar da reconhecida exigência da representatividade populacional no delineamento de estudos epidemiológicos, há uma escassez das publicações discutindo procedimentos éticos e eficazes para a captação de participantes.

O presente trabalho tem como objetivo apresentar os motivos de recusa entre idosos selecionados para um estudo populacional de acompanhamento longitudinal.

\section{Método}

Foi conduzido um estudo qualitativo, baseado na abordagem de análise de conteúdo proposta por Laurence Bardin, ${ }^{6}$ para analisar o motivo da recusa de idosos convidados para participar da segunda fase do estudo Fragilidade em Idosos Brasileiros - Seção-RJ (FIBRA-RJ).

\section{O estudo FIBRA}

O estudo Fragilidade em Idosos Brasileiros (FIBRA) 


\section{Artigo original}

foi idealizado pelo consórcio de quatro universidades brasileiras (Universidade do Estado do Rio de Janeiro, Universidade de São Paulo-Ribeirão Preto, Universidade Federal de Minas Gerais e Universidade Estadual de Campinas), e teve seu projeto aprovado no final de 2006 pelo Conselho Nacional de Desenvolvimento Científico e Tecnológico (CNPq), sob o processo 555087/2006-9, sendo aprovado pelo Comitê de Ética e Pesquisa do Hospital Universitário Pedro Ernesto (1850-CEP/HUPE). A partir do referido estudo, o polo da Universidade do Estado do Rio de Janeiro constituiu a Rede FIBRA-RJ, no período entre janeiro de 2009 a janeiro de 2010, com o objetivo de determinar a prevalência e investigar as características da síndrome de fragilidade da cidade do Rio de Janeiro, quando foram entrevistados 847 idosos, clientes de uma operadora de saúde, residentes na Zona Norte do município do Rio de Janeiro.,8

A partir das informações obtidas nesta linha de base, foi conduzido o estudo de seguimento com o propósito de ampliar a investigação dos aspectos cognitivos da população estudada. Especificamente, nesta segunda fase de investigação, conduzida no período de julho de 2010 a junho de 2011, a amostra suspeita de declínio cognitivo foi submetida à avaliação clínica e neuropsicológica com o objetivo de se identificar a presença de síndrome demencial.Foram incluídos neste estudo de seguimento 683 idosos investigados na linha de base. Essa etapa de pesquisa recebeu financiamento da Fundação de Amparo à Pesquisa do Estado do Rio de Janeiro (FAPERJ), Processo E-26 110.294/2007, e foi aprovada pela Comissão de Ética em Pesquisa da SubReitoria de Pós-Graduação e Pesquisa da Universidade do Estado Rio de Janeiro (016.3.2008-COEP 027/2008).

\section{Participantes do presente estudo}

Para recrutar a amostra para o estudo de seguimento que objetivou estimar a prevalência de demência em idosos da Zona Norte da cidade do Rio de Janeiro, partiu-se do contato com todos os idosos cujo escore do MiniExame do Estado Mental (MEEM) ${ }^{9,10}$ foi inferior a 28 pontos na primeira onda. Dentre os idosos que participaram do estudo, 546 apresentaram o referido escore no teste. Adicionalmente, entre os indivíduos com pontuação superior ou igual a 28 no MEEM, considerados preservados cognitivamente, foi realizado um sorteio para que uma amostra aleatória (20\%) destes indivíduos ( $n=51$ ) pudesse ser avaliada na etapa de seguimento.

Por meio de recrutamento telefônico, familiares destes idosos eram convidados a responder ao questionário de atividades funcionais de Pfeffer (FAQ). ${ }^{11,12}$
Aqueles que atingiram MEEM inferior a 28 pontos e FAQ inferior a cinco pontos $(n=486)$ e mais os 51 idosos sorteados no grupo preservado cognitivamente foram convidados para uma avaliação complexa composta por avaliação clínica e neuropsicológica, exames laboratoriais e de imagem voltados para o diagnóstico de síndrome demencial. ${ }^{13}$

A captação desses indivíduos resultou em um total de 67 recusas, que correspondem a 7,9\% da amostra inicial e quase $20 \%$ do total suspeito de síndrome demencial. Esse grupo não participante da segunda fase do estudo FIBRA-RJ corresponde à população de estudo da presente investigação.

\section{Procedimentos para recrutamento da amostra}

Um rigoroso treinamento de auxiliares de pesquisa foi realizado para recrutar potenciais participantes, em que se teve o cuidado de selecionar apenas aqueles que demonstraram habilidades em contato telefônico e estavam completamente afinados com a proposta de trabalho. Todas as informações eram detalhadas ao entrevistado, inclusive abordando as etapas seguintes, deixando claro que todos os exames seriam realizados sem nenhum custo e que, caso fosse de interesse do idoso ou da família, seria oferecido, sem nenhum custo, o tratamento no ambulatório de geriatria da universidade. No entanto, observou-se que muitos idosos ou acompanhantes recusavam participar do estudo. Todos os motivos de recusa foram armazenados com a finalidade de estudá-los ao final do trabalho.

\section{Referencial teórico-metodológico}

Para o tratamento das informações relativas às pessoas que recusaram participar da pesquisa foi utilizada a análise de conteúdo do tipo categorial temática. A análise de conteúdo diz respeito a uma técnica sistemática de investigação da mensagem, desenvolvida nos Estados Unidos, num contexto em que prevaleciam os estudos behavioristas nas ciências humanas, com a finalidade de identificar, por meio de meios de comunicação de massa (imprensa e rádio), as tendências políticas de outros países. Em poucas palavras, ela consiste num conjunto de técnicas que auxiliam a análise de comunicações. O uso da análise de conteúdo pressupõe o abandono da ideia de que há transparência nos fatos sociais, uma vez que consiste em selecionar temas que se repetem a fim de identificar uma estrutura subjacente ao que é dito. ${ }^{6}$

Silva e colaboradores ${ }^{14}$ retomam Bardin ${ }^{6}$ para men- 
cionar duas funções da análise de conteúdo: 1) função heurística de exploração e probabilidade de descoberta de sentidos subjacentes; 2) administração de comprovações a partir de hipóteses que norteiam a análise.

A análise de conteúdo pode se valer tanto de técnicas qualitativas como da quantitativa. Contudo, há diferenças na maneira como se tomam os dados. A análise qualitativa é utilizada nos lançamentos de hipóteses, enquanto que o aspecto quantitativo obteria dados descritivos por meio do uso da estatística, servindo, portanto, para verificar hipóteses. ${ }^{6}$

Os estudos qualitativos se tornam particularmente úteis quando o objeto a ser estudado ainda não foi explorado pela comunidade científica, pois auxiliam no conhecimento de aspectos ainda não avaliados do problema a ser investigado, além de aprofundar essa investigação. Na pesquisa no âmbito da saúde, o método qualitativo auxilia na compreensão do significado individual ou coletivo dos fenômenos associados ao processo de saúde-doença, possibilitando o entendimento de como as pessoas se organizam diante do adoecimento e também na prevenção e no cuidado com a saúde..$^{15}$

A análise de dados de uma pesquisa de campo costuma oferecer alguns obstáculos: primeiro, o fato de que os pesquisadores podem incorrer numa "ilusão da transparência" ao crer que, a partir dos dados, o contexto real se mostra de modo claro a eles; segundo, existe o perigo de ocorrer um apego demasiado à técnica que deixe de lado a busca primeira da pesquisa: o significado do material coletado; terceiro, a dificuldade de aplicar teorias e conceitos abstratos em demasia ao que foi coletado. Por esses motivos, a análise de material objetiva a descoberta a partir da própria pesquisa, partindo de hipóteses provisórias que a levem a outras de modo a ampliar o contexto de significados investigados. ${ }^{16}$

O método para tratamento do conteúdo se dá em três momentos: 1) pré-análise, 2) exploração do material e 3) tratamento dos resultados, inferência e interpretação. A pré-análise envolve um momento de organização em que há a escolha dos documentos a serem submetidos à formulação de hipóteses e objetivos, e aos indicadores que fundamentam a interpretação final. A exploração do material nada mais é que o tratamento sistemático das decisões já tomadas. Por fim, há a interpretação dos resultados já obtidos, às vezes, utilizando porcentagens ou análise fatorial. Provas, estatísticas e testes de validação são meios de estabelecer maior rigor e, ao encontrar resultados significativos e fiéis, o analista pode fazer inferências e interpretações de acordo com os objetivos previstos. ${ }^{6}$

Existem alguns tipos de análise de conteúdo, como análise de avaliação, das relações, de enunciação, de expressão e categorial temática. Esta última costuma ser o tipo mais comum ${ }^{6,17}$ e foi utilizada para a análise das recusas no presente estudo.

Esse método consiste na classificação de elementos constitutivos por diferenciação e, posteriormente, por reagrupamento a partir das diferenças temáticas, por exemplo, ou por aproximação entre assuntos. Há, basicamente, duas etapas na categorização: 1) inventário - nesta, os elementos são isolados; e 2) classificação - procurar colocar uma organização às mensagens.

A análise temática, desta maneira, identifica os núcleos de sentido que envolvem a comunicação. Nesta, a frequência com que um tema aparece costuma apontar para a relevância deste assunto na mensagem analisada. Há, porém, variações ao considerar a intensidade e expressão de alguns itens. Meireles e Cendón ${ }^{17}$ observam que as inferências obtidas a partir das categorias respondem pela identificação das questões de maior relevância contidas no texto.

É importante dizer que a análise de conteúdo e a análise documental se diferenciam em alguns pontos, como: 1) trabalha com mensagens (comunicação); 2) seus objetivos são diferentes: enquanto a primeira trabalha com o conteúdo e a expressão destes, visando a identificação de uma realidade subjacente à mensagem, a análise documental objetiva sintetizar as informações em classificações com vistas à consulta e armazenagem.

\section{Procedimentos para a análise de dados}

O conteúdo analisado se compôs do registro feito pelo recrutador a cada contato com os idosos e/ou seus informantes ao longo do recrutamento.

Os conteúdos das conversas foram descritos pelos auxiliares de pesquisa no momento do contato ao telefone, realizado para recrutar os particpipantes elegíveis com o objetivo de manter um histórico que permitisse ao próximo recrutador conhecer a situação. Com frequência, era necessário mais de um contato com cada participante para se concluir a situação de aceite ou recusa da participação no estudo. O material de análise foi constituído, portanto, pelos registros das falas em todos os contatos feitos com os indivíduos que se recusaram a participar.

Num primeiro momento destacamos os principais motivos de recusa. Posteriormente, os conteúdos foram categorizados em recusa do informante (familiar, cuidador ou pessoa próxima indicada pelo idoso) e recusa 


\section{Artigo original}

do próprio idoso. Dentre estas, foram criados os núcleos figurativos com os principais motivos de recusa.

\section{Resultados}

Foram analisadas as falas de 67 idosos $(7,9 \%$ da amostra inicial), dentre os quais $73 \%$ do sexo feminino. A média de idade foi de 79,22 (DP =6,71) anos.

Os núcleos figurativos criados para descrever os motivos de recusa foram: presença de doença, falta de credibilidade no estudo, desconfiança, falta de tempo, ausência de um informante e distância. No quadro 1 , foram apresentados exemplos de cada núcleo figurativo obtido na análise de conteúdo.

Foram obtidas 53,7\% de recusas por parte dos próprios idosos e $46,3 \%$ por parte do informante. Na figura 1 foi descrita a frequência dos núcleos figurativos criados, que explicam os principais motivos de recusas. Observase que a falta de credibilidade na pesquisa e a doença foram as principais causas de recusa nesta amostra, representando 53,7\% e 14,9\% dos motivos, respectivamente. A falta de credibilidade e a desconfiança foram os motivos mais frequentes entre os próprios idosos e a falta de tempo, o acompanhamento médico vigente e a doença os mais frequentes entre os informantes.

Executar projetos de pesquisa populacional não

Quadro 1. Exemplos retirados das falas dos participantes da pesquisa e categorizados conforme os núcleos figurativos.

\begin{tabular}{|c|c|}
\hline Núcleos figurativos & Exemplos \\
\hline Falta de credibilidade & $\begin{array}{l}\text { "Toda pesquisa é uma perda de tem- } \\
\text { po, uma palhaçada (...)." }\end{array}$ \\
\hline Desconfiança & $\begin{array}{l}\text { "Recentemente três amigas sofreram } \\
\text { um golpe por telefone". }\end{array}$ \\
\hline Doença & $\begin{array}{l}\text { "A idosa não tem condições de falar } \\
\text { ao telefone devido ao Alzheimer (...), } \\
\text { se ela começasse a falar, iria se perder } \\
\text { e mudar de assunto e no final acabar- } \\
\text { ia tendo que falar com ele mesmo." }\end{array}$ \\
\hline Falta de tempo & $\begin{array}{l}\text { "Filho disse que estava com um aluno } \\
\text { e não poderia falar naquele momen- } \\
\text { to. Disse que tinha que dar aula e que } \\
\text { isso era mais importante para ele do } \\
\text { que marcar a consulta da mãe." }\end{array}$ \\
\hline $\begin{array}{l}\text { Acompanhamento médico } \\
\text { vigente }\end{array}$ & $\begin{array}{l}\text { "Já faz tratamento com um neurolo- } \\
\text { gista." }\end{array}$ \\
\hline Falta de informante & $\begin{array}{l}\text { "Não tem ninguém disponível no } \\
\text { momento." }\end{array}$ \\
\hline Distância & $\begin{array}{l}\text { "Resistência da parte do idoso e da } \\
\text { filha por causa da distância, por de- } \\
\text { penderem do carro do marido de M. e } \\
\text { porque ela cuida de seus três filhos." }\end{array}$ \\
\hline
\end{tabular}

é tarefa fácil. Requer habilidade do recrutador e um tempo grande tentando um argumento convincente para a participação do indivíduo. As recusas são responsáveis por grande parte das perdas nos estudos epidemiológicos e a não adesão da amostra selecionada aleatoriamente pode significar o comprometimento da validade externa dos achados obtidos. Geralmente, o sujeito - que é parte fundamental - não tem consciência do seu papel neste processo.

Nos estudos na área da saúde, as recusas na linha de base e ao longo do seguimento podem levar a viés de seleção e, consequentemente, a estimativas tendenciosas, por exemplo, à subestimação de prevalências e das medidas de associação quando a não participação se deve à exposição e ao desfecho de interesse. ${ }^{18,19}$ No presente estudo, identificou-se a falta de credibilidade em pesquisas e a presença de doenças como os principais motivos para recusa em participar do estudo. Este último consiste em fator que está associado aos agravos de saúde em populações mais velhas que, na maioria das vezes, representa a relevância e o próprio objeto de estudo de pesquisadores da área gerontológica. Assim, a questão central de pesquisa, quando associada ao adoecimento, pode ser um dos maiores desafios para a captação de participantes idosos. Nesse sentido, prever estratégias de orientação, encaminhamento e contrapartidas capazes de atender às demandas que surgirão decorrentes da presença de patologias na população de estudo, além de um compromisso ético da pesquisa em saúde, pode contribuir para o aumento da adesão dos participantes aos procedimentos do estudo. Além disso, cientistas sociais apontam, entre as estratégias para melhor condução das pesquisas em gerontologia, o uso de uma equipe de investigação multicultural; a manutenção de boas relações com os líderes comunitários de forma a se garantir familiaridade com a cultura local; e ainda a parceria e comunicação aberta entre os cientistas interessados em um mesmo tema. ${ }^{20}$

Dentre as causas de recusa relatadas na literatura, Waldman e colaboradores ${ }^{21}$ argumentam que a exigência do termo de consentimento assinado pelo entrevistado pode contribuir para o aumento desnecessário das perdas nos estudos populacionais, principalmente, quando o estudo envolve indivíduos de baixa escolaridade. Como é comum no caso de investigações com coortes mais velhas no Brasil, a exigência de assinatura representa uma natural desconfiança entre analfabetos que não conseguem analisar o conteúdo do documento, desta forma essa exigência está associada, na prática, 
ao aumento das perdas e recusas. Esses mesmos autores apontam o quanto são tênues os mecanismos de convencimento dos entrevistadores e que, diante ausência de riscos nos procedimentos de coleta de dados, a maior preocupação ética é quanto à confidencialidade. Assim, a garantia de sigilo e a revisão da exigência do termo de consentimento assinado poderiam ser empregadas a favor da diminuição das recusas nas entrevistas realizadas em domicílio.

No presente estudo, o recrutamento para participação foi realizado por telefone, o que reduziu a dificuldade com o consentimento assinado, não obstante esse método de captação apresentou suas próprias dificuldades, como a necessidade de diversos telefonemas com o mesmo indivíduo para conseguir a adesão ao estudo. No Brasil, o Ministério da Saúde vem realizando inquéritos telefônicos, por meio do sistema Vigilância de Fatores de Risco e Proteção para Doenças Crônicas por Inquérito Telefônico (VIGITEL), nos quais são mantidas baixas taxas de recusas $(9,1 \%$ das linhas elegíveis no inquérito de 2006 e 3,0\% em 2010), sendo contabilizadas cerca de nove ligações por entrevista completa. ${ }^{21}$ Segundo pesquisadores do VIGITEL, uma estratégia eficaz para diminuir as recusas foi permitir a interrupção da entrevista, se necessário, e o agendamento para continuá-la em ocasião conveniente para o participante. Para incentivar a participação dos indivíduos sorteados foram apontados os mecanismos de esclarecer que as perguntas não eram de natureza pessoal, garantir o anonimato e o direito de não participação.

Outra estratégia amplamente utilizada para reduzir os problemas de recusas consiste na divulgação do estudo junto à população-fonte, como ocorre nos censos realizados pelo IBGE, que apresentam baixas frequências de não respostas. ${ }^{21}$

Entre um dos principais motivos de recusa relatados pelo familiar do idoso, no presente estudo, esteve a "falta de tempo". A dificuldade decorrente da falta de disponibilidade para participar da pesquisa pode ser facilmente resolvida com estratégias de ampliação dos turnos de entrevistas em horários não comerciais e nos fins de semana. Contudo, este argumento, quando apresentado por familiares de idosos, pode ser resultado de uma falta de envolvimento ou sobrecarga de cuidadores informais com as demandas do cuidado e tratamento de saúde do idoso. Na fala destes familiares apareceram argumentações como não ter necessidade de tratamento ou ainda relatos de que a condição de saúde do idoso era tão grave que desacreditava-se na pesquisa como estratégia que faria qualquer diferença. Esta última argumentação pode estar associada à falta de credibilidade na pesquisa e no sistema de saúde, em que predominam a crença de que a participação individual não possa fazer diferença a nível coletivo, isto é, que a estratégia dos estudos observacionais não promove mudanças na gestão e na prática clínica em saúde.

A presente investigação, a partir da análise qualitativa do argumento de idosos que recusaram a con-

Figura 1. Frequência dos núcleos figurativos.

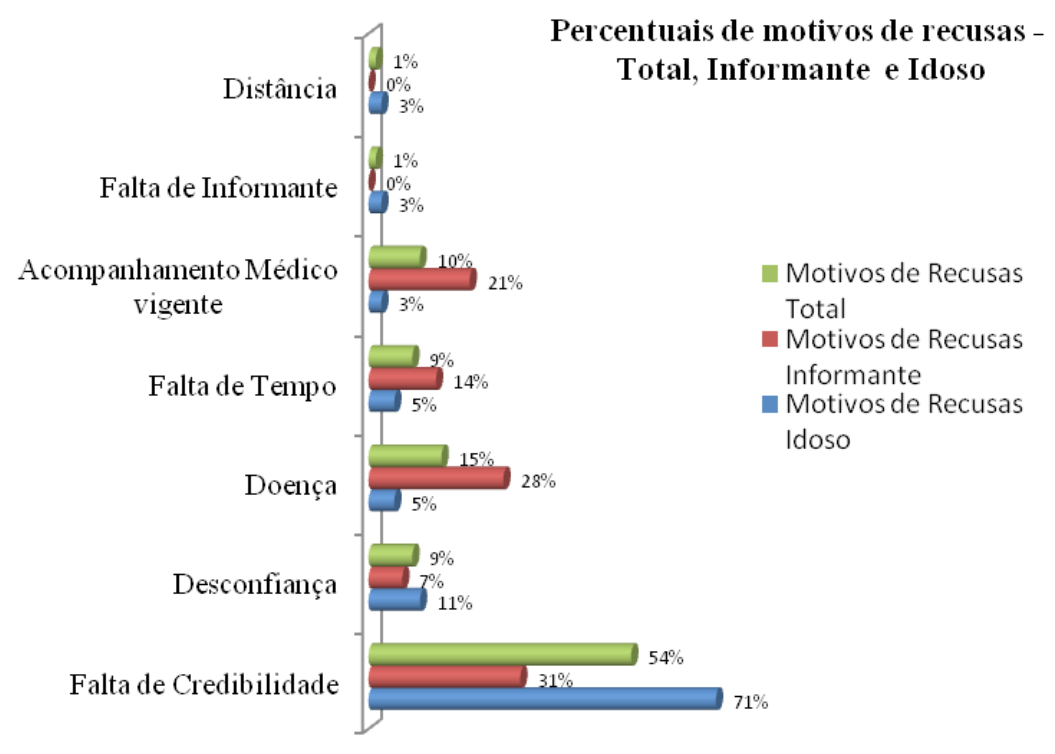




\section{Artigo original}

tinuidade como participantes de uma pesquisa com escopo em saúde mental do idoso, pretende divulgar e refletir sobre estratégias de planejamento que ampliem a captação e representatividade da população-alvo em estudos epidemiológicos. Conclui-se que a condição de saúde do participante e a desconfiança acerca da pesquisa são os principais argumentos para recusa. Assim, na condução de pesquisas com a população idosa, pode ser útil reconhecer as demandas deste grupo e elaborar e divulgar estratégias de suporte aos cuidados das condições de saúde investigadas. Reduzir o distanciamento dos resultados de pesquisa e o cotidiano da população geral pode contribuir para uma mudança cultural quanto ao significado de ser participante de um estudo científico e do quanto a investigação científica pode impactar a prática em saúde coletiva.

\section{Agradecimentos}

À FAPERJ, o financiamento do Estudo FIBRA-RJ.

\section{Referências}

1. Instituto Brasileiro de Geografia e Estatística (IBGE). Projeção da População do Brasil por sexo e idade: 1980-2050. Revisão 2008. Est. \& Pesq., 2008 [acesso em 12 set 2015]. Disponível em: http://www.ibge.gov.br/home/estatistica/populacao/projecao_da_popula-cao/2008/projecao.pdf

2. Chaimovicz F, Camargos MCS. Envelhecimento e Saúde no Brasil. In: Freitas EV, Py L, organizadores. Tratado de Geriatria e Gerontologia. Rio de Janeiro: Guanabara Koogan; 2011. p.74-98.

3. Pinto RBR, Bastos LC. Approach to research in epidemiology applied to gerontology in Brazil: literature review of papers published between 1995 and 2005. Rev Bras Epidemiol. set 2007;(3):361-9.

4. Rosset I, Pedrazzi EC, Roriz-Cruz M, et al. Tendencies of studies addressing the eldest individuals of aged population in the community: a (inter)national systematic review. Rev Esc Enferm. USP [online] 2011;45(1):264-71.

5. Brasil. Resolução 466/2012. Diretrizes e normas regulamentadoras de pesquisas envolvendo seres humanos. Ministério da Saúde/Conselho Nacional de Saúde, Brasília, 12 dez. 2012. [acesso em 12 Set 2015]. Disponível em: http://conselho. saude.gov.br/ resolucoes/2012/Reso466.pdf

6. Bardin L. Análise de conteúdo. Lisboa: Edições 70; 1977.

7. Perez M, Lourenço RA. FIBRA-RJ Network: frailty and risk of hospitalization in the elderly in Rio de Janeiro, Brazil. Cad Saude Publica. 2013;29(7):1381-91.

8. Moreira VG, Lourenço RA. Prevalence and factors associated with frailty in an older population from the city of Rio de Janeiro, Brazil: the FIBRA-RJ Study. Clinics. 2013;68(7):979-85.

9. Folstein MF, Folstein SE, Mchuch PR. "Mini-mental state". A practical method for grading the cognitive state of patients for the clinician. J Psychiatr Res. 1975;12(3):189-98.

10. Lourenço RA, Veras RP. Mini-Mental State Examination: psychometric characteristics in elderly outpatients. Rev Saude Publica. 2006;40(4):712-9.

11. Pfeffer RI, Kurosaki TT, Harrah CH Jr, et al. Measurement of functional activities in older adults in the community. Journal Gerontol. 1982;37(3):323-9.

12. Sanchez MAS, Correa PCR, Lourenço RA. Cross-cultural Adaptation of the "Functional Activities Questionnaire - FAQ" for use in Brazil. Dementia \& Neuropsychologia. 2011;5(4):322-7.

13. Ribeiro PCC, Lopes CS, Lourenço RA. Prevalence of Dementia in Elderly Clients of a Private Health Care Plan: a Study of the FIBRA-RJ, Brazil. Dement Geriatr Cogn Disord. 2013;35:77-86.

14. Silva $C R$, Gobbi BC, Simão AA. O uso da análise de conteúdo como uma ferramenta para a pesquisa qualitativa: descrição e aplicação do método. Organizações rurais \& agroindustriais. 2005;7(1):70-81.

15. Turato ER. Qualitative and quantitative methods in health: definitions, differences and research subjects. Rev Saude Publica. 2005 Jun;39(3):507-14. Epub 2005 Jun 30.

16. Minayo MCS. O desafio do conhecimento: pesquisa qualitativa em saúde. São Paulo-Rio de Janeiro: Hucitec-Abrasco; 1992. p. 197-211.

17. Meireles MRG, Cendón BV. Aplicação prática dos processos de análise de conteúdo e de análise de citações em artigos relacionados às Redes Neurais Artificiais. Informação \& Informação. 2010;15(2):77-93.

18. Kristman V, Manno M, Côté P. Loss to Follow-Up in Cohort Studies: How Much is Too Much? Eur J Epidemiol. 2004;19(8):751-60.

19. Coeli CM, Carvalho MS, Chor D, et al. Cad Saude Colet. 2012;20(1):102-5.

20. Chi I. Cross-cultural gerontology research methods: challenges and solutions. Ageing Soc. 2011;31:371-85.

21. Ministério da Saúde, Secretaria de Vigilância em Saúde, Secretaria de Gestão Estratégica e Participativa Vigitel Brasil 2009: vigilância de fatores de risco e proteção para doenças crônicas por inquérito telefônico. Brasília: Ministério da Saúde; 2010.

22. Waldman EA, Novaes HMD, Albuquerque, MFM, et al. Inquéritos populacionais: aspectos metodológicos, operacionais e éticos. Rev Bras Epidemiol. 2008;11(supl 1):68-79. 